\title{
Young isolated neutron stars from the Gould Belt
}

\author{
S. B. Popov ${ }^{1,4}$, M. Colpi ${ }^{2}$, M. E. Prokhorov ${ }^{1}$, A. Treves ${ }^{3}$, and R. Turolla ${ }^{4}$ \\ 1 Sternberg Astronomical Institute, Universitetski pr. 13, 119992 Moscow, Russia \\ e-mail: mike@sai .msu.ru \\ 2 Università di Milano-Bicocca, Dipartimento di Fisica, Piazza della Scienza 3, 20126, Milano, Italy \\ e-mail: Monica.Colpi@mib.infn.it \\ 3 Università dell'Insubria, Dipartimento di Scienze, via Vallegio 11, 22100, Como, Italy \\ e-mail: treves@mib.infn.it \\ ${ }^{4}$ Università di Padova, Dipartimento di Fisica, via Marzolo 8, 35131, Padova, Italy \\ e-mail: turolla@pd.infn.it; popov@pd.infn.it
}

Received 27 November 2002 / Accepted 2 April 2003

\begin{abstract}
The origin of the local population of young, cooling neutron stars is investigated with a population synthesis model taking into account the contribution of neutron stars born in the Gould Belt, in addition to those originating in the Galactic disk. We estimate their emission in the soft X-ray band as a function of distance and age and construct the $\log N-\log S$ distribution. It is shown that the inclusion of neutron stars from the Gould Belt provides a good fit to the observed Log $N-$ $\log S$ distribution. As the Sun is situated inside the Gould Belt, one can naturally explain the comparative local overabundance of massive progenitors and can remove the difficulty of the deficit of relatively bright $\left(\approx 0.1\right.$ ROSAT PSPC cts s $\left.{ }^{-1}\right)$ cooling neutron stars previously reported from models where only neutron stars from the Galactic disk were accounted for.
\end{abstract}

Key words. stars: neutron - stars: evolution - stars: statistics - X-rays: stars

\section{Introduction}

Observations of isolated neutron stars (INSs) are important for gaining deeper insight on their structure and thermal evolution and ultimately might prove decisive in unveiling the physical properties of matter at ultra-high densities. Up to a decade ago, the only known INSs were active radio pulsars, with the addition of the $\gamma$-ray pulsar Geminga. Despite the fact that X-ray emission from some radio pulsars was already detected by Einstein, it was in the 90's that ROSAT, thanks to its sensitivity in the $0.1-2 \mathrm{keV}$ band, gave a clearer picture of the faint $\mathrm{X}$-ray emission produced by the cooling surface of the closest INSs. ROSAT, supplemented by more recent observations by Chandra and XMM-Newton, revealed a variety of types of behavior in the X-ray emission from INSs and its relation with radio activity.

In particular, a substantial contribution of ROSAT to this field has been the discovery of a group of seven radioquiet, close-by, thermally-emitting INSs, dubbed ROSAT INSs (RINSs) and sometimes referred to as the "magnificent seven" (see for recent references Treves et al. 2000; Zampieri et al. 2001; Haberl \& Zavlin 2002; see also Popov \& Prokhorov 2003). The nature of these sources has been controversial and two interpretations were proposed, either as conventional middle-aged cooling NSs or as very old NSs accreting the

Send offprint requests to: S. B. Popov, e-mail: polar@sai.msu.ru interstellar gas (see again Treves et al. 2000). Although no compelling evidence has been brought in favor of either picture as yet, it is now generally believed that at least two of these objects (the brightest ones, RX J1856-3754 and RX J0720.4-3125, e.g. Braje \& Romani 2002) are indeed relatively young cooling NSs.

Since the seven RINSs have remarkably similar observed properties, it is quite natural to assume that they belong to the same class, i.e. all of them are close-by, cooling NSs. This, however, poses a major problem. A useful and standard way to study a population of sources is to compute the $\log N-\log S$ distribution starting from a model, and then compare it with observations. This has been done for RINSs by Neuhäuser \& Trümper (1999) and Popov et al. (2000b). The main conclusion of the latter investigation is that the typical spatial density of radio pulsars in our Galaxy is too low to explain RINSs. In other words, the assumption that RINSs and ordinary radio pulsars derive from the same parent population underpredicts the number of observed "coolers", i.e. NSs which are hot and close enough for their thermal emission to be detected in X-rays.

An obvious solution is to invoke a local (both in space and time) overabundance of NSs with respect to those originating in the Galactic disk and seen now as radio pulsars. The main goal of this paper is to investigate the possible origin of these objects. Here we suggest that the likely birthplace for many of the INSs in the Solar proximity is the Gould Belt (see 
Popov et al. 2002 for a preliminary discussion). The Gould Belt is a compound of young stellar associations extending in a ring tilted at about $20^{\circ}$ from the Galactic plane, roughly centered at the position of the Sun and with a radius of $\sim 1 \mathrm{kpc}$. Originally discovered in the middle of the 19th century, the Belt was studied in detail by Benjamin Gould (see Stothers \& Frogel 1974 for a historical overview). Most of the known close-by young star associations (in Ophiuchus, Orion, Perseus, Scorpius etc.) belong to the Belt. Since the Gould Belt is a relatively young stellar system it contains a comparatively large fraction of massive stars. As the Solar proximity is embedded within the Belt, it is likely to have harbored quite a number of massive young stars which are the progenitors of NSs.

In Sect. 2 we perform a population synthesis of close-by young INSs, assuming that they are born both in the Gould Belt and in the Galactic disk with an assigned mass spectrum. The dynamical evolution of the young NSs population in the Galactic potential is then followed together with the thermal evolution. This allows us to compute the present surface temperature and spatial distributions of young, close-by NSs and finally their X-ray count rate, once a model for interstellar absorption has been prescribed. This is used to compute the $\log N$-Log $S$ distribution which is then compared with observations in Sect. 3. We refer to ROSAT PSPC in evaluating the X-ray count rate and in confronting our model with data. Even if Chandra and XMM-Newton provide much better observations of individual sources and small fields, ROSAT all-sky data are still the most complete by far in soft X-rays. Discussion and conclusions follow in Sect. 4.

\section{The model}

In this section we describe the method we used to compute the local distribution of young, cooling neutron stars. The main ingredients of our model are: the spatial distribution of NS progenitors, the NS formation rate, the NS cooling history, and the interstellar medium (ISM) distribution (the latter determines the interstellar absorption and hence affects the X-ray count rate). Even if we are concerned with a young population ( $\tau \lesssim 10^{6}$ yrs), its dynamical evolution can not be neglected if the number of sources in a limited volume (size $\lesssim 1 \mathrm{kpc}$ ) has to be assessed. In fact, a NS with a typical velocity $\sim 300 \mathrm{~km} \mathrm{~s}^{-1}$ travels a distance $\sim 300 \mathrm{pc}$ in its lifetime as a cooler. While not much on a Galactic scale, such a displacement is non-negligible in evaluating the cooler population in the Solar proximity. For this reason we account for the dynamical evolution of NSs in the Galactic potential in our model.

Our calculation proceeds in three steps. First, a spatial distribution of the progenitors is selected and the ratio of NSs born in the Galactic plane to those originating from the Gould Belt is fixed, together with the birth rates (Sect. 2.1). Second, the dynamical evolution is followed assuming that NSs at birth receive a kick velocity drawn from a prescribed velocity distribution (see Sect. 2.2). Finally, we derive the X-ray flux as a function of age and position on the basis of an updated set of cooling curves (Sect. 2.3), and translate this in ROSAT count rate for an assumed model of the interstellar absorption (Sect. 2.4).

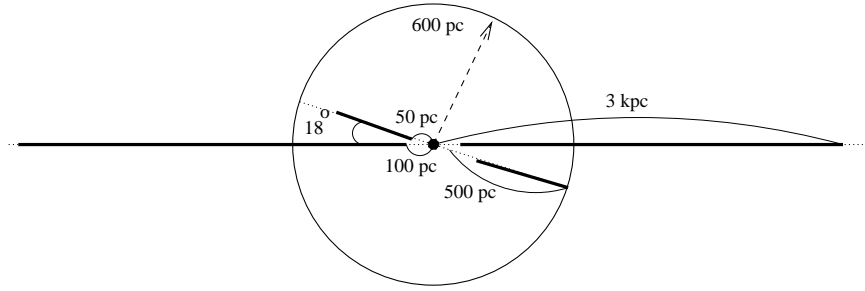

Fig. 1. $R-z$ projection of the initial spatial distribution of NSs. The heavy lines mark the Gould Belt and the closer $(<3 \mathrm{kpc})$ part of the Galactic disk where NSs are assumed to be born. Note the two regions devoid of NS formation, in the Galactic disk with a radius of $100 \mathrm{pc}$ around the Sun, and in the Belt with a radius $150 \mathrm{pc}$ around its center. The $0.6 \mathrm{kpc}$ sphere centered at the Sun is also shown for clarity.

We varied the parameters of our model (NS formation rate, NSs mass spectrum, time of calculation, time step, spatial distribution). In the following subsections we focus on those parameters corresponding to the results presented in Sect. 3.

\subsection{Initial spatial distribution}

In our picture NSs are born in the Galactic disk and in the Gould Belt with a constant rate over the entire calculation (see Fig. 1 for a schematic view). This is a reasonable approximation since we are dealing with young objects, less than a few Myrs old. The Gould Belt is modeled as a very thin disk of $500 \mathrm{pc}$ in radius and inclination to the Galactic plane of $18^{\circ}$ with its center situated at $100 \mathrm{pc}$ from the Sun in the Galactic anticenter direction. The central region of the Gould Belt is devoid of massive stars (see Pöppel 1997 for detailed description of the Gould Belt, and Torra et al. 2000 for a shorter one). To mimic this, we assume that no NS is born in the central region of the Belt up to a distance of $150 \mathrm{pc}$ from its center.

We assume that the birthrate per unit area is independent of position, i.e. it is constant both in the Belt and in the Galactic disk. The two (constant) values are different, the one referring to the Belt being larger. To derive the NS birthrate, we rely upon direct counting of massive stars which are doomed to end in a supernova event. This gives $2.9 \times 10^{-11}$ massive progenitors per yr per $\mathrm{pc}^{2}$ (Tammann et al. 1994) which implies a NS birthrate of $30 \mathrm{NSs} \mathrm{Myr}^{-1}$ in $0.6 \mathrm{kpc}$ around the Sun, the region where data are available. In relating the NS and supernova birthrates we assumed that core collapse produces a neutron star in $90 \%$ of the cases. The above value gives a more reliable estimate of the local NS birthrate, at present, as compared with radio pulsar and average supernova statistics.

To get the separate contributions to the NS birthrate from the Belt and from the disk for the region inside $0.6 \mathrm{kpc}$ we proceed as follows. In one Myr, out of a total of thirty, twenty NSs are born in the Gould Belt according to the estimate of 20-27 supernova events given by Grenier (2000). The remainder is uniformly distributed in the Galactic disk in a ring extending from 100 to $600 \mathrm{pc}$, to account for the known fact that the Solar neighbourhood is underpopulated by massive stars (e.g. Maíz-Apellániz 2001). This is in agreement with the results of Torra et al. (2000), who estimate that about $2 / 3$ of the massive stars inside $0.6 \mathrm{kpc}$ belong to the Gould Belt, 
and the rest to the Galactic disk. Direct counting of massive progenitors within $0.6 \mathrm{kpc}$ from the Sun accounts for all NSs originating in the Belt. However, the disk clearly extends well beyond $0.6 \mathrm{kpc}$ from the Sun. Although NSs born far away are very dim their contribution may become significant at low fluxes $\left(\lessgtr 0.1 \mathrm{cts} \mathrm{s}^{-1}\right)$. For this reason we decided to include also NSs born in the Galactic disk from 0.6 up to $3 \mathrm{kpc}$ from the Sun. The NS birthrate per unit area in this region is assumed to be again constant and coincident with that in the disk inside $0.6 \mathrm{kpc}$. With this choice we find an agreement with existing data on the supernova rate in a region of $1 \mathrm{kpc}$ around the Sun (Tammann et al. 1994).

In summary, we adopt a uniform birthrate of $20 \mathrm{NS} \mathrm{Myr}^{-1}$ in the Gould Belt and $250 \mathrm{NSs} \mathrm{Myr}^{-1}$ in the Galactic plane up to a limiting distance of $3 \mathrm{kpc}$ from the Sun. Our approach underestimates the number of distant ( $\gtrsim 1 \mathrm{kpc}$ ) newborn NSs in the direction of the Galactic center because the rate of supernova events is likely to increase there. However, interstellar absorption towards the Galactic center is very high in the Galactic plane (see Sect. 2.4), and this hinders the detection of sources at low Galactic latitude. We note that the local overabundance of young NSs is probably due not only to the enhanced number of massive stars harbored in the Belt but reflects also the fact that the star formation history in the Belt favors an enhanced supernova rate in the present epoch.

\subsection{Dynamical evolution}

A detailed description of the evolutionary code may be found in Popov et al. (2000a,b). Typically we calculate about a thousand evolutionary tracks in each run (up to $\sim 10^{4}$ per production run) and then normalize our results to the actual number of NSs born in the volume $(\sim 1000)$ during the 4.25 Myrs time interval, as discussed above. An evolutionary track is calculated for specified initial position and velocity of a NS. All tracks are used over their whole duration and are applied to NSs of different masses, i.e. for each track we have several different cooling histories, the number of which depends on the number of different masses used in the run. As in Popov et al. (2000a,b), each track actually represents a population of NSs of different masses continuously born at the specified location with a prescribed spatial velocity distribution, during the entire time interval of the calculation.

NSs are assumed to be born with a Maxwellian kick velocity distribution with an average of $\sim 225 \mathrm{~km} \mathrm{~s}^{-1}$. Varying this parameter does not change our results significantly because we are probing young objects. The Galactic potential is assumed to be axisymmetric, resulting from the sum of three contributions: the disk, bulge and halo (see Paczynski 1990). The Sun is in the Galactic plane at a distance $8.5 \mathrm{kpc}$ from the Galactic Center.

\subsection{Cooling curves and flux calculation}

To calculate the cooling of NSs we use the results of the St. Petersburg group (see Kaminker et al. 2002, and the review by Yakovlev et al. 1999). Cooling curves are for NSs

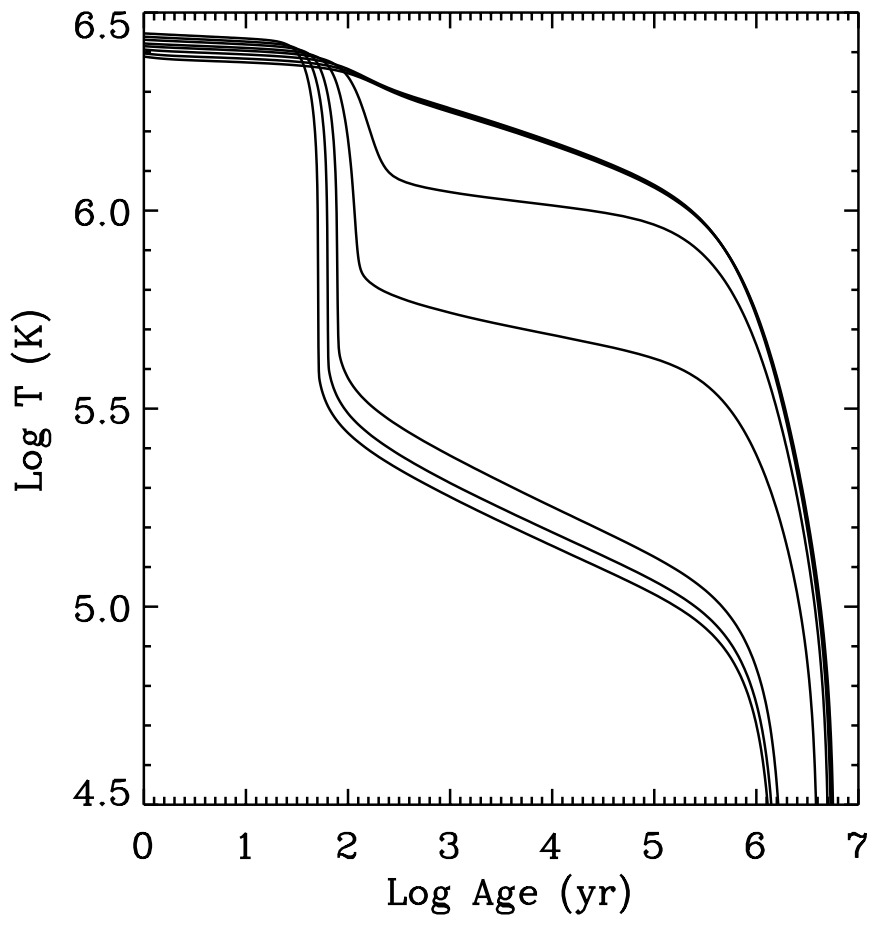

Fig. 2. Cooling curves for different NS masses (Kaminker et al. 2002). Curves from top to bottom correspond to masses 1.1-1.8 $M_{\odot}$ (step $\left.0.1 M_{\odot}\right)$. Here, and everywhere in the text, the temperature is the redshifted surface temperature (i.e. that observed at infinity).

of masses from $1.1 M_{\odot}$ to $1.8 M_{\odot}$ with a step of $0.1 M_{\odot}$ (see Fig. 2). Curves take into account all important processes of neutrino emission. The equation of state (EOS) used in Kaminker et al. (2002) was introduced by Prakash et al. (1988). More precisely, it corresponds to Model I of Prakash et al. for symmetry energy and compression modulus of saturated nuclear matter $K=240 \mathrm{MeV}$. The maximum NS mass in this model is $1.977 M_{\odot}$. Since we base our calculation on a definite EOS, the star radius $R$ is known once the mass $M$ is fixed. For $1.1 M_{\odot}<M<1.8 M_{\odot}$ it is $12.2 \mathrm{~km}<R<13.2 \mathrm{~km}$, where $R$ is circumferential radius. The EOS used here corresponds to matter composed of neutrons, protons, and electrons (no muons, hyperons, and no exotic particles). Neutron superfluidity was ignored.

We start to compute the emitted flux when a NS has an age of $10000 \mathrm{yrs}$. This choice is motivated by the fact that the Vela pulsar (the youngest close-by thermally emitting NS) is slightly older than that and also because the NS birth rate used in our calculation corresponds to an event every $\sim 10000$ yrs in the $\sim 1.7 \mathrm{kpc}$ region around the Sun. For each NS the calculation is stopped when the temperature drops below $10^{5} \mathrm{~K}$. This happens at an age of 4.25 Myrs for the lightest NSs $\left(M=1.1 M_{\odot}\right)$ or less for more massive stars. NSs that cold could have been detected by ROSAT PSPC within a distance of only $10 \mathrm{pc}$ with a count rate $\sim 5 \times 10^{-3} \mathrm{cts} \mathrm{s}^{-1}$. We assume that emission comes from the entire star surface. This appears reasonable in the light of the relatively low pulsed fractions $(\$ 15-20 \%)$ detected so far in the majority of isolated NSs (see Haberl \& Zavlin 2002). 
We do not take into account any reprocessing of the blackbody surface emission in the NS atmosphere.

NSs are expected to have a mass spectrum (see discussion in Woosley et al. 2002). In the calculations we present here NSs are taken to have a flat mass spectrum between 1.1 and $1.8 M_{\odot}$. The mass spectrum (and clearly the mass of each star) are assumed to be constant during the calculations. With the above values (maximum age 4.25 Myrs and time step $10000 \mathrm{yrs}$ ) each evolutionary track represents $425 \times 8 \mathrm{NSs}$ of eight different masses born with a time step $10^{4}$ yrs at the same place with the same initial velocity in a period 4.25-0.01 Myrs ago.

As one can see from Fig. 2, the main contribution to X-ray bright sources comes from NSs with masses $<1.5 M_{\odot}$. Young isolated NSs which are observed in several $\mathrm{SN}$ remnants as compact X-ray sources also should be relatively low-mass objects (Kaminker et al. 2002). Observations of binary radio pulsars suggest that NSs masses are strongly peaked around $1.35 M_{\odot}$ (Thorsett \& Chakrabaty 1999). We repeated our calculations using a similar distribution and found that it produces similar results, although the number of observable isolated NSs increases by $\sim 30 \%$. Calculations which take into account the realistic mass function of massive progenitors in the Solar vicinity will be the subject of a future paper.

\subsection{ISM and absorption}

Since young cooling NSs are expected to emit most of their luminosity at UV/soft X-ray energies ( 20-200 eV, corresponding to temperatures $\sim 10^{5}-10^{6} \mathrm{~K}$ ), interstellar absorption plays a crucial role with respect to their observability. Any attempt to derive the number of observable cooling isolated NSs using the unabsorbed flux would result in a substantial overestimate.

For the ISM distribution we use the same prescription as in Popov \& Prokhorov (1998). The Local Bubble is modeled as a sphere with a radius of $140 \mathrm{pc}$ and ISM density of $0.1 \mathrm{~cm}^{-3}$. Typical column densities for sources inside the calculated volume are in the range $N_{\mathrm{H}} \sim 10^{19}-10^{21} \mathrm{~cm}^{2}$. After the column density is evaluated for a current NS position, we calculate the unabsorbed flux corresponding to the temperature, radius of the NS and its distance from the Sun, and apply the standard procedure to derive the ROSAT PSPC count rate. Outside the Local Bubble in directions close to the Galactic plane, absorption starts to play a crucial role. That is why regions closer to the Galactic center $(<7 \mathrm{kpc})$, where the NS formation rate should be higher than in the Galactic disk in the Solar vicinity, cannot add many sources to our sample. At $N_{\mathrm{H}}=3 \times 10^{21} \mathrm{~cm}^{-2}$ even very young and hot low-mass NSs with $k T \approx 0.1 \mathrm{keV}$ cannot have been detected at a distance $1 \mathrm{kpc}$ in the ROSAT Bright Survey (RBS) at the threshold of $\sim 0.2 \mathrm{cts} \mathrm{s}^{-1}$.

Except for the Local Bubble (and even that in a quite simplified way, see Sfeir et al. 1999 for a more complete description), our model does not take into account small-scale irregularities in the ISM distribution. They can be important if one makes an attempt to produce a realistic map of RINSs distribution on the celestial sphere, but in the case of the all-sky averaged $\log N-\log S$ distribution our approximation is adequate.

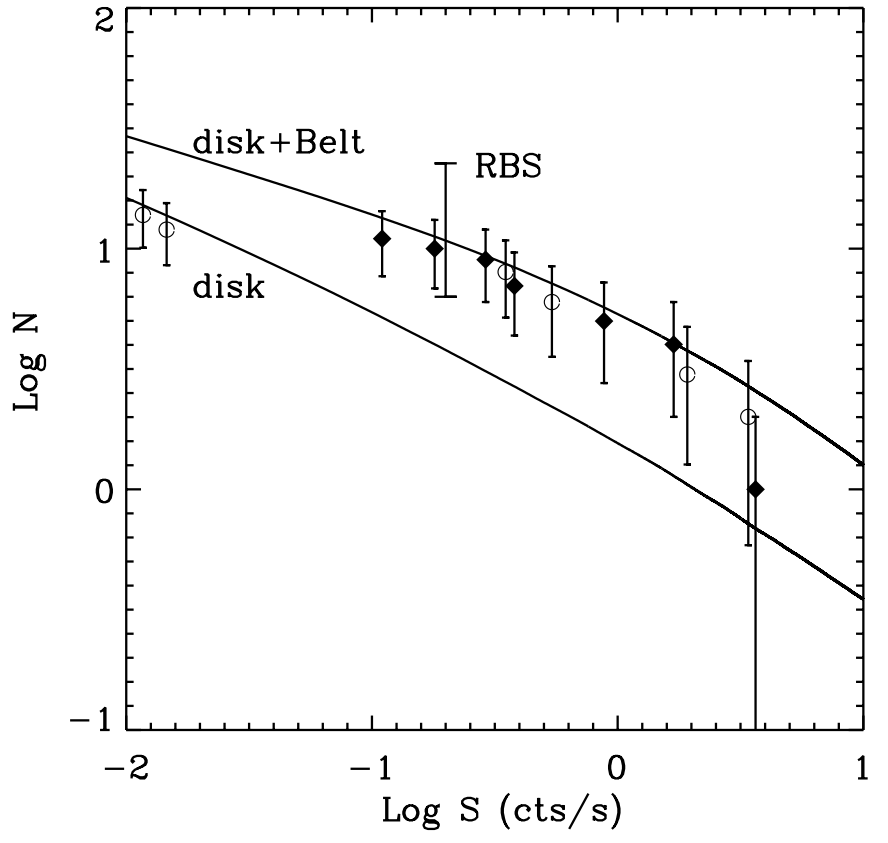

Fig. 3. All-sky $\log N-\log S$ distribution: filled diamonds are the seven RINSs and open circles Geminga, the "three musketeers", PSR 1929+10 and 3EG J1835+5918. We also show the RBS limit (Schwope et al. 1999). Upper curve: NSs born in the Gould Belt and in the Galactic disk (total birth rate $270 \mathrm{NS} \mathrm{Myr}^{-1}$ ). Lower curve: disk only (birth rate $250 \mathrm{NS} \mathrm{Myr}^{-1}$ ).

\section{Results}

As discussed in the previous section, the dynamical evolution of young neutron stars, together with the calculation of their Xray flux, allows us to derive the $\log N-\log S$ for these sources. In particular, having fixed the number of NSs originating in the Gould Belt and in the Galactic disk, we can compute the separate contributions of these two sub-populations to the total $\log N-\log S$. Our main results are presented in Fig. 3 where the total (disk+Belt) and the disk-only distributions are compared with observations. All curves refer to the whole sky, i.e. the angular coverage used here is the entire solid angle. The observed $\log N-\log S$ has been derived from ROSAT data of the seven RINSs and six other close-by young isolated NSs (see Table 1). Contrary to RINSs, the latter sources exhibit a composite spectrum with a non-thermal high-energy tail superimposed on the thermal component. The total count rate has been used in this case, but we stress that the non-thermal contribution is sizeable only for the Vela pulsar and PSR 1929+10. Poissonian errors are assumed in both observations and simulations. The computed curves were generated with a sufficiently large number of stars to reduce the statistical noise which is particularly severe at large flux (see Sect. 2.2). Statistical errors are shown for the observational points only, being too small to be appreciated in the plot of the simulated distributions. Uncertainties arising from the assumed model parameters are more difficult to assess and could introduce non-negligible deviations. Test runs with different choices of key parameters show that differences are of the order of $50 \%$. Symbols which show observational points (filled diamonds or open circles) 
Table 1. Local $(r<1 \mathrm{kpc}$ ) population of young (age $<4.25$ Myrs) isolated neutron stars.

\begin{tabular}{|c|c|c|c|c|c|c|}
\hline Source name & $\begin{array}{c}\text { Period, } \\
\mathrm{s}\end{array}$ & $\begin{array}{c}\text { Count rate, } \\
\text { ROSAT cts s }^{-1}\end{array}$ & $\begin{array}{c}\dot{P} \\
10^{-15} \mathrm{ss}^{-1}\end{array}$ & $\begin{array}{c}\text { Distance } \\
\mathrm{kpc}\end{array}$ & $\begin{array}{l}\mathrm{Age}^{a} \\
\text { Myrs }\end{array}$ & Ref. \\
\hline RX J185635-3754 & - & 3.64 & - & $0.117^{d}$ & $\sim 0.5$ & {$[1,2]$} \\
\hline RX J0720.4-3125 & 8.37 & 1.69 & $\sim 30-60$ & - & - & {$[1,3]$} \\
\hline 1RXS J130848.6+212708 (RBS 1223) & 10.3 & 0.29 & $<10^{4} ?$ & - & - & {$[1,4]$} \\
\hline RX J1605.3+3249 (RBS 1556) & - & 0.88 & - & - & - & {$[1]$} \\
\hline RX J0806.4-4123 & 11.37 & 0.38 & - & - & - & {$[1,5]$} \\
\hline RX J0420.0-5022 & 22.7 & 0.11 & - & - & - & [1] \\
\hline 1RXS J214303.7+065419 (RBS 1774) & - & 0.18 & - & - & - & {$[6]$} \\
\hline PSR B0633+17 (Geminga) & 0.237 & $0.54^{c}$ & 10.97 & $0.16^{d}$ & 0.34 & [7] \\
\hline RX J1836.2+5925 (3EG J1835+5918) & - & 0.015 & - & - & - & {$[8]$} \\
\hline PSR B0833-45 (Vela) & 0.089 & $3.4^{c}$ & 124.88 & $0.294^{d}$ & 0.01 & {$[7,9,10]$} \\
\hline PSR B0656+14 & 0.385 & $1.92^{c}$ & 55.01 & $0.762^{e}$ & 0.11 & {$[7,10]$} \\
\hline PSR B1055-52 & 0.197 & $0.35^{c}$ & 5.83 & $\sim 1^{b}$ & 0.54 & {$[7,10]$} \\
\hline PSR B1929+10 & 0.227 & $0.012^{c}$ & 1.16 & $0.33^{d}$ & 3.1 & {$[7,10]$} \\
\hline PSR J0056+4756 & 0.472 & - & 3.57 & $0.998^{e}$ & 2.1 & {$[10]$} \\
\hline PSR J0454+5543 & 0.341 & - & 2.37 & $0.793^{e}$ & 2.3 & {$[10]$} \\
\hline PSR J1918+1541 & 0.371 & - & 2.54 & $0.684^{e}$ & 2.3 & [10] \\
\hline PSR J2048-1616 & 1.962 & - & 10.96 & $0.639^{e}$ & 2.8 & {$[10]$} \\
\hline PSR J1848-1952 & 4.308 & - & 23.31 & $0.956^{e}$ & 2.9 & [10] \\
\hline PSR J0837+0610 & 1.274 & - & 6.8 & $0.722^{e}$ & 3.0 & [10] \\
\hline PSR J1908+0734 & 0.212 & - & 0.82 & $0.584^{e}$ & 4.1 & {$[10]$} \\
\hline
\end{tabular}

${ }^{a}$ Radio pulsar ages are estimated as $P /(2 \dot{P})$,

for RX J1856-3754 the age estimate comes from kinematical considerations (Walter \& Lattimer 2002).

${ }^{b}$ Distance to PSR 1055-52 is uncertain $(\sim 0.9-1.5 \mathrm{kpc})$

${ }^{c}$ Total count rate (blackbody + non-thermal)

${ }^{d}$ Distances from parallactic measurements

${ }^{e}$ Distances from the dispersion measure

(1) Treves et al. (2000), (2) Kaplan et al. (2002), (3) Zane et al. (2002), (4) Hambaryan et al. (2001), Haberl (2003), (5) Haberl \& Zavlin (2002), (6) Zampieri et al. (2001), (7) Becker \& Trümper (1997), (8) Mirabal \& Halpern (2001), (9) Pavlov et al. (2001), (10) ATNF Pulsar Catalogue, (http://wwwatnf.atnf.csiro.au/research/pulsar/catalogue/)

correspond to the type of the faintest object (RINS or not) which contributes to the total number at the specified count rate.

Previous investigations have convincingly shown that cooling NSs with the same spatial density of ordinary radio pulsars cannot explain the observed Log $N-\log S$ distribution for RINSs (Neuhäuser \& Trümper 1999; Popov et al. 2000b). In particular, it was stressed that an additional contribution to the local population of young cooling NSs should be invoked to explain the relatively large number of bright sources at fluxes $\gtrsim 0.1 \mathrm{cts} \mathrm{s}^{-1}$. The Gould Belt is a natural candidate to provide the missing NSs in the Solar proximity. The population synthesis calculations presented here strongly support this claim and account not only for the observed distribution of RINSs but also for that of other young, close-by isolated NSs observed by ROSAT.

As can be seen from Fig. 3, the Gould Belt provides the major contribution to the local population of young cooling NSs and the theoretical prediction gives a very good fit to the data, within statistical errors. The contribution of NSs born in the Galactic disk is not very important at relatively large fluxes (it leaves room for only a few sources with count rate $\gtrsim 0.1 \mathrm{cts} \mathrm{s}^{-1}$ ), but, as expected, becomes dominant at lower fluxes $\left(\lessgtr 0.01 \mathrm{cts} \mathrm{s}^{-1}\right)$ where far away stars contribute most. Figure 3 clearly shows that the curve referring to the Galactic disk alone is always below the observed points with high statistical significance and below the RBS limit. The worsening of the agreement at low fluxes $\left(\lessgtr 0.05 \mathrm{cts} \mathrm{s}^{-1}\right)$ is to be attributed to the incompleteness of the X-ray sample. The deficit of very bright objects ( $\gtrsim 1 \mathrm{cts} \mathrm{s}^{-1}$ ) may be attributed to chance statistics.

Our calculations show that there can be about 10-50 unidentified isolated NSs in the ROSAT All-Sky Survey (RASS) at a limiting flux of $\gtrsim 0.015 \mathrm{cts} \mathrm{s}^{-1}$ depending on parameters of the model. The number of INSs in the RASS/BSC has been recently investigated by Rutledge et al. (2003), who found that at most 67 sources could have been detected at the 0.05 cts s$^{-1}$ level and have escaped identification. Our results are well within this limit. Also there may 
be a few unidentified RINSs at fluxes $\gtrsim 0.1$ cts s$^{-1}$ at low Galactic latitudes (see also Schwope et al. 1999). Most sources should be observed at $\pm 20^{\circ}$ from the Galactic plane towards the directions of lower absorption. Some of them may be Geminga-like objects with counterparts among unidentified gamma-ray sources (also connected with the Gould Belt, see Grenier 2000). Identification of these objects may prove important for constraining cooling models and the NS mass spectrum.

Absorption, the flat geometry of NS initial distribution and the finite extension of the Gould Belt naturally explain the very flat (slope flatter than -1) $\log N-\log S$ curves in Fig. 3. Our model contains some degrees of freedom (e.g. the NS mass spectrum, details of the formation rate and surface emission, a simple blackbody was used here) that can be varied. We nevertheless believe that even this simple picture (albeit based on realistic assumptions) gives a quite satisfactory explanation of the observed properties of isolated NSs in the Solar proximity. Preliminary calculations which take into account realistic mass spectrum of NS progenitors, atmospheric effects and different spatial and velocity distributions are in progress and show no qualitative changes.

\section{Discussion and conclusions}

At present about 20 nearby (distance $<1 \mathrm{kpc}$ ), young (age $<4.25$ Myrs) isolated NSs are known (see Table 1). The local NS population includes objects with different properties: radio-quiet, thermally-emitting NSs (the seven RINSs), Geminga and the Geminga-like object 3EG J1835+5918 (these are probably active pulsars with radiobeams missing the Earth), radio pulsars with observed thermal X-ray emission (PSR 1929+10 and the "three musketeers": the Vela pulsar, PSR 0656+14, PSR 1055-52) and seven other pulsars which are not detected in X-rays. The latter objects are relatively old in comparison with the others and lie further away, as can be seen from Table 1 where the wide gap in the estimated age of PSR 1055-52 and PSR J0056+4756 is apparent. For ages of a few Myrs, even very low-mass (and hence slow-cooling) NSs are too cold by now to have been detected with ROSAT at distances $\gtrsim 0.5 \mathrm{kpc}$. The case of PSR $1929+10$ is intermediate since it is $\sim 3$ Myrs old but relatively close $(\sim 300 \mathrm{pc})$ and its $\mathrm{X}$-ray emission is mainly non-thermal.

As has been discussed by Neuhäuser \& Trümper (1999) and Popov et al. (2000b), the number of X-ray bright INSs is too large to be explained in terms of the average density of radio pulsars in the Solar neighborhood. This motivated the suggestion that a sizeable fraction of NSs never become active radio emitters, as suggested at the same time by considerations on young NSs in supernova remnants (Gotthelf \& Vasisht 2000) albeit the origin of the local NS population was left open. In this paper we have shown that thermally-emitting INSs are naturally explained as cooling NSs born in the Gould Belt. The relatively high local spatial density of young NSs is then due to the large number of massive progenitors in the young stellar associations which constitute the Gould Belt. Our analysis lends support to the idea that RINSs (the "magnificent seven") represent the slowly cooling NSs and hence a clean sample of NSs with $M \lesssim 1.3 M_{\odot}$ (Kaminker et al. 2002).

The computed $\log N$-Log $S$ distribution for X-ray thermally-emitting INSs born in the Gould Belt and (to a lesser extent) in the Galactic disk accounts for all bright INSs in the Solar vicinity (see Fig. 3) and leaves room for at most 1-2 undetected sources above $\sim 1 \mathrm{cts} \mathrm{s}^{-1}$. The absence of sources brighter than RX J1856 $(\log S>0.56)$ is consistent with our calculations at the $2 \sigma$ level. Having neglected very young NSs (age $<10000$ yrs) does not change our conclusions significantly. Only young stars with $M<1.35 M_{\odot}$ (about $1 / 2$ of their total number) have temperatures about $2 \times 10^{6} \mathrm{~K}$ (see Fig. 2), which corresponds to a luminosity $\sim 10^{34} \mathrm{erg} \mathrm{s}^{-1}$. With our NS formation rate we expect to have one such NS within a distance of $\sim 2.5 \mathrm{kpc}$. About half of the NSs born in that region should originate inside $1.7 \mathrm{kpc}$. Placing the source at this distance its unabsorbed flux is $\sim 3 \times 10^{-11} \mathrm{erg} \mathrm{cm}^{-2} \mathrm{~s}^{-1}$. With a typical column density of $10^{21} \mathrm{~cm}^{-2}$, Web-PIMMS gives $\sim 1.3 \mathrm{cts} \mathrm{s}^{-1}$ for ROSAT PSPC. We can then conclude that stars younger than 10000 yrs can contribute only to fluxes $<1.3 \mathrm{cts} \mathrm{s}^{-1}$. This translates into a $\sim 20 \%$ difference in the $\log N-\log S$ curve shown in Fig. 3 in the worst case. Typical uncertainties of the present model are at about the same level.

The local ( $\$ 1 \mathrm{kpc}$ ) INS population was estimated to comprise about one hundred objects younger than $\sim 4$ Myr, taking into account that some NSs born inside $1 \mathrm{kpc}$ can leave the local space in their lifetime. These NSs are not detected as radio pulsars, but tens of them could be identified in ROSAT surveys as dim sources. The beaming effect can be responsible only for part of these young NSs to be radiosilent. According to Tauris \& Manchester (1998) in fact, about 50-70\% of young pulsars are not visible from the Earth (see also Brazier \& Johnston 1999). In our model this would imply the presence of at least $\sim 30$ active pulsars with an age $\$ 4$ Myrs in the Solar vicinity. However, only about $1 / 3$ of such pulsars are observed (see again Table 1). A bimodal velocity distribution, with the high characteristic velocity $\gtrsim 500 \mathrm{~km} \mathrm{~s}^{-1}$ (Arzoumanian et al. 2002), would reduce the discrepancy since a number of young pulsars leave the local volume considered here. However, even barring observational bias against low-luminosity radio pulsars, our model seems to support the argument by Gotthelf \& Vasisht (2000), that at least half of the observed young neutron stars follow an evolutionary path quite distinct from that of the Crab pulsar.

Acknowledgements. We want to thank Dmitry Yakovlev for putting his cooling model at our disposal and for his invaluable help with it. We are also indebted to Vasily Beskin, Matteo Chieregato and Andrea Possenti for many useful discussions.The work of MP was supported by the Russian Foundation for Basic Research (RFBR) grant 03-02-16068. The work of SP was supported by the Russian Foundation for Basic Research (RFBR) grant 02-02-06663 and by RSCI. SP thanks the Universities of Insubria at Como and MilanoBicocca, where part of this investigation was carried out, for hospitality. The work of MC, AT and RT was partially supported by the Italian Ministry for Education, University and Research (MIUR) under grant COFIN-2000-MM02C71842. 


\section{References}

Arzoumanian, Z., Chernoff, D. F., \& Cordes, J. M. 2002, ApJ, 568, 289

Becker, W., \& Trümper, J. 1997, A\&A, 326, 682

Braje, T. M., \& Romani, R. W. 2002, ApJ, 580, 1043

Brazier, K. T. S., \& Johnston, S. 1999, MNRAS, 305, 671

Gotthelf, E. V., \& Vasisht, G. 2000, in Proc. of IAU Coll. 177, Pulsar astronomy - 2000 and beyond, ed. M. Kramer, N. Wex, \& N. Wielibinski, ASP Conf. Ser., 202, 699

Grenier, I. A. 2000, A\&A, 364, L93

Haberl, F., \& Zavlin, V. E. 2002, A\&A, 391, 571

Haberl, F. 2003, COSPAR Symp. on High Energy Studies of Supernova Remnants and Neutron Stars, in press [astro-ph/0302540]

Hambaryan, V., Hasinger., G., Schwope, A. D., \& Schulz, N. S. 2001, A\&A, 381, 98

Kaminker, A. D., Yakovlev, D. G., \& Gnedin, O. Yu. 2002, A\&A, 383, 1076

Kaplan, D. L., van Kerkwijk, M. H., \& Anderson, J. 2002, ApJ, 571, 447

Maíz-Apellániz, J. 2001, AJ, 121, 2737

Mirabal, N., \& Halpern, J. P. 2001, ApJ, 547, L137

Neuhäuser, R., \& Trümper, J. 1999, A\&A, 343, 151

Paczynski, B. 1990, ApJ, 348, 485

Pavlov, G. G., Zavlin, V. E., Sanwal, D., Burwitz, V., \& Garmire, G. P. 2001, ApJ, 552, L129

Pöppel, W. 1997, Fund. Cosm. Phys., 18, 1

Popov, S. B., Colpi, M., Treves, A., et al. 2000a, ApJ, 530, 896
Popov, S. B., Colpi, M., Prokhorov, M. E., Treves, A., \& Turolla, R. 2000b, ApJ, 544, L53

Popov, S. B., Prokhorov, M. E., Colpi, M., Treves, A., \& Turolla, R. 2002, Grav. \& Cosmol., 8 (suppl. II), 133, [astro-ph/0201030]

Popov, S. B., \& Prokhorov, M. E. 1998, A\&A, 331, 535

Popov, S. B., \& Prokhorov, M. E. 2003, Trudy GAISH, in press [astro-ph/0205298]

Prakash, M., Ainsworth, T. L., \& Lattimer, J. M. 1988, Phys. Rev. Lett., 61, 2518

Rutledge, R. E., Fox, D. W., Bogosavljevic, M., \& Mahabal, A. 2003, ApJ, in press [astro-ph/0302107]

Schwope, A. D., Hasinger, G., Schwarz, R., Haberl, F., \& Schmidt, M. 1999, A\&A, 341, L51

Sfeir, D. M., Lallement, R., Crifo, F., \& Welsh, B. Y. 1999, ApJ, 346, 785

Stothers, R., \& Frogel, J. A. 1974, AJ, 79, 456

Tammann, G. A., Löffler, W., \& Schröder, A. 1994, ApJS, 92, 487

Tauris, T. M., \& Manchester, R. N. 1998, MNRAS, 298, 625

Thorsett, S. E., \& Chakrabarty, D. 1999, ApJ, 512, 288

Torra, J., Fernández, D., \& Figueras, F. 2000, A\&A, 359, 82

Treves, A., Turolla, R., Zane, S., \& Colpi, M. 2000, PASP, 112, 297

Walter, F. M., \& Lattimer, J. M. 2002, ApJ, 576, L145

Woosley, S. E., Heger, A., \& Weaver, T. A. 2002, Rev. Mod. Phys., 74, 1015

Yakovlev, D. G., Levenfish, K. P., \& Shibanov, Yu. A. 1999, Phys. Uspekhi, 42, 737

Zampieri, L., Campana, S., Turolla, R., et al. 2001, A\&A, 378, L5

Zane, S., Haberl, F., Cropper, M., et al. 2002, MNRAS, 334, 345 